BCS70 (collected at age 10). Adult psychological well-being (NCDS=age 33; BCS70=age 30) was indicated by psychological ill health and self-efficacy. Adult psychological ill health was indicated by the Malaise Inventory with a cutoff point of 7 or above. Selfefficacy was derived from the response to questions asking the study participants about their perceived level of control over their life.

Methods A dichotomised index of childhood adversity was created after tabulating information about parenthood, mother's age, mother's education, and presence of older siblings. The effect of breast feeding on childhood psychosocial adjustment and adult psychological well-being was examined using logistic regression. Men and women were analysed separately and the effects of breast feeding on the outcomes were adjusted for confounders.

Results Findings showed that the magnitude of the effect of breast feeding on adult psychological well-being is larger in women than in men. After accounting for the effect of childhood social adversity, breast feeding promoted psychosocial adjustment during childhood in girls in NCDS (OR 1.25, 95\% CI 1.05 to 1.48) and in BCS70 (OR $1.44,95 \%$ CI 1.15 to 1.81 ), but not in boys.

In adulthood, being breast fed at birth was associated with higher self-efficacy (OR 1.31, 95\% CI 1.06 to to 1.61) and lower risk of psychological ill health (OR $0.76,95 \%$ CI 0.61 to to 0.96 ) in women in BCS70 only. However, no significant interaction effect was found between breast feeding and childhood social adversity.

Conclusion Although breast feeding did not moderate the negative effect of childhood social adversity on childhood or adulthood outcomes in this study, findings suggest that the practice of breast feeding can be important for women's psychological well-being throughout the lifecourse.

\section{BREAST FEEDING AND BEHAVIOURAL DEVELOPMENT IN CHILDREN: FINDINGS FROM THE MILLENNIUM COHORT STUDY}

\section{doi:10.1136/jech.2010.120956.12}

${ }^{1} \mathrm{~K}$ Heikkilä, ${ }^{2} \mathrm{~A}$ Sacker, ${ }^{3} \mathrm{Y}$ Kelly, ${ }^{4} \mathrm{M} \mathrm{J}$ Renfrew, ${ }^{1} \mathrm{M}$ A Quigley. ${ }^{1}$ National Perinatal Epidemiology Unit, University of Oxford, UK; ${ }^{2}$ Institute for Social and Economic Research, University of Essex, UK; ${ }^{3}$ Department of Epidemiology and Public Health, University College London, UK; ${ }^{4}$ Mother and Infant Research Unit, University of York, UK

Aim Our aim was to examine whether breast feeding is associated with behavioural development in children aged 5 years.

Methods We used data from a large, prospective, nationally representative UK cohort, the Millennium Cohort Study. Breast feeding was ascertained from parent-interviews at baseline and child behaviour from parent-rated outcome, the Strengths and Difficulties Questionnaire (SDQ). Our analyses included 10037 mother-child pairs with data on breast feeding, SDQ and potential confounders. 9525 of the children were born at term and 512 were preterm. We used logistic regression models to investigate the associations of breast feeding duration with abnormal parent-rated SDQ total and sub-scores at age five in term and preterm children separately.

Results Overall, abnormal SDQ scores were less common in breast fed than formula-fed children. Term children breast fed for four months or longer had lower odds of an abnormal total SDQ score (multivariable-adjusted $\mathrm{OR}$ compared to never breast fed children: $0.65,95 \%$ CI 0.52 to 0.82 ). This effect was similar for all the SDO sub-scores. In preterm children prolonged breast feeding was generally associated with lower odds of abnormal SDQ total and sub-scores but the effect estimates were imprecise. The associations between exclusive breast feeding and abnormal SDQ scores were similar to those of any breast feeding and abnormal SDQ scores.

Conclusions Our findings suggest that, at least in term children, prolonged breast feeding is associated with having fewer parentrated behavioural problems at the age of 5 years.

\section{(Un)employment and health}

\section{HAS YOUR WORK WORKED YOU TOO HARD: AN EXAMINATION OF WORK HISTORY, PRESENT FUNCTIONAL LIMITATIONS AND REDUCED ACTIVITIES OF LIVING IN A COHORT OF THE IRISH GENERAL POPULATION}

doi:10.1136/jech.2010.120956.13

V J C Mc Carthy, I J Perry, B A Greiner. Department of Epidemiology and Public Health, University College Cork, Cork, Republic of Ireland

Objective In the present paper, we examine the association between manual work, resultant functional limitations and reduced ability to carry out daily activities of living (ADL), in older age. We hypothesise that manual workers as opposed to non-manual workers suffer, in older age, functional limitations and reduced ability to carry out ADL. This study is of great importance owing to our ageing Irish population and an ailing health service.

Methods A 10 year follow up study was conducted on a cohort of the general population (59-80 year olds) in the Republic of Ireland. Specific data on physical measurements, marital status, educational attainment, work history, functional status and $\mathrm{ADL}$ was collected on 357 study participants. Work history data focused on the job the participant had done for the longest period of time, paid or unpaid. Each participant was then asked if they described this work as manual or non-manual. Functional limitations and ADL were assessed using validated scales.

Results Just over half the sample were female (53\%) with $44 \%$ $(n=150)$ retired (median $=9$ (5.14) years). Over $60 \%$ of the participants were, or had been engaged in manual work with this percentage higher in males, albeit non-significant (68\% vs $58 \%$, $\mathrm{p}=0.08$ ). $20 \%$ of the total sample had complete function with a higher proportion of non-manual as opposed to manual workers $(p=0.07)$ with no limitations. Almost three quarters of the sample had functional limitations and less than one fifth were classified as having an $\mathrm{ADL}$ disability.

Using linear regression, manual workers were significantly more likely to have functional limitations even after adjustment $(B=0.85$, $\mathrm{SE}=0.30, \mathrm{p}=0.01)$ for socio-demographic factors. When stratified by age, manual work remained significantly associated with functional limitations in the 60-69 year olds $(B=0.66, S E=0.34, p=0.05)$ and in the $70-80$ year olds $(B=1.13, S E=0.50, p=0.03)$. For the older age group, males had a decreased risk of functional limitations independent of work type $(B=-1.06, S E=0.47, p=0.03)$.

There was no significant association between manual work and reduced activities of daily living either in the unadjusted or adjusted model.

Discussion Functional limitations, in an older population, are related to the type of work they carried out. Good health surveillance for manual workers at a young age can identify limitations early. Initiatives such as work organisation, education and promotion of best work practices with regard to manual work can reduce functional limitations in older age.

\section{HOW DO MUSCULOSKELETAL PAIN, AVOIDANT COPING AND SICKNESS ABSENCE RELATE TO EACH OTHER?}

\section{doi:10.1136/jech.2010.120956.14}

U Christensen, C Ørsted Hougaard, K Thielen, E Nygaard, R Lund. Department of Public Health, Section of Social Medicine, University of Copenhagen

Background Musculoskeletal pain is common and causes extensive sickness absence and work disability in many industrialised countries. The prevalence of musculoskeletal morbidity increases by decreasing socio-economic position and it appears that people with a disadvantaged social situation are more vulnerable to the 
consequences of musculoskeletal disorders. An avoidant coping strategy has been associated with an increased likelihood of sickness absence, and some evidence has been shown of increasing use of avoidant coping with decreasing social class.

Objectives The objective of this study is to examine the single and joint effect of musculoskeletal pain and avoidant coping on sickness absence and how this effect may be moderated by socio-economic position.

Methods This study was based on a prospective design including survey data from 2000 and 2006 and register data from 2007. The study population consists of a sample of Danes in their 40s and 50s free of major depression at baseline and in 2006, economically active in 2006, and reporting functional limitations due to musculoskeletal pain, $N=2967$. The outcome measure was retrieved from Statistics Denmark and contained information on sickness absence $>2$ weeks in 2007. By multivariate logistic regression the association between self-reported musculoskeletal pain (daily vs weekly/monthly/ seldom and never) and sickness absence was studied, adjusted by avoidant coping, physical exposures in work environment, gender and socio-economic position (measured by occupational social class). The joint effect of pain and avoidant coping was calculated as departure from multiplicativity and tested by product terms.

Results The adjusted OR between musculoskeletal pain and sickness absence was 1.76 (95\% CI 1.44 to to 2.15). Further analysis showed a departure from multiplicativity for the joint effect of pain and avoidant coping on sickness absence. Socio-economic position had a strong independent effect on the risk of sickness absence $(p<0.0001)$. However, there was no significant moderating effect of socio-economic position on the joint effect of pain and avoidant coping.

Conclusion An avoidant coping strategy interacts with the perception of pain and has a strong effect on the risk of sickness absence. Although socio-economic position is significantly associated with the risk of sickness absence it did not moderate the joint effect of avoidant coping and musculoskeletal pain.

\section{PATHWAYS TO WORK? INSIGHTS FROM A SYSTEMATIC REVIEW OF THE UK'S RETURN TO WORK INITIATIVES FOR DISABLED AND CHRONICALLY ILL PEOPLE}

doi:10.1136/jech.2010.120956.15

${ }^{1} \mathrm{~S}$ Clayton, ${ }^{1} \mathrm{R}$ Gosling, ${ }^{1} \mathrm{~S}$ Povall, ${ }^{2} \mathrm{~K}$ Misso, ${ }^{3} \mathrm{C}$ Bambra, ${ }^{1} \mathrm{M}$ Whitehead. ${ }^{1}$ Division of Public Health, University of Liverpool, UK; ${ }^{2}$ Centre for Reviews and Dissemination, University of York, UK; ${ }^{3}$ Wolfson Research Institute, Durham University, UK

Purpose Employment rates of chronically ill and disabled people in the UK are low (49\%) and 2.7 million are on disability-related state benefits. Recent government policy has seen the introduction of a range of measures aimed at integrating them into the labour market. This paper explores what can be learned for future policy from a synthesis of a wide range of evidence evaluating these policies.

Method We conducted a systematic review and evidence synthesis of empirical studies reporting the employment effects and/or factors influencing the effectiveness of national-level interventions aimed at helping people into work who were not employed and were on some form of disability-related benefit. We excluded measures aimed at reducing short-term sickness absence, localised interventions, and those that were not wholly concerned with helping individuals move into the open labour market.

Results In total, 42 studies were identified that met the inclusion criteria and were included in the full review. This paper synthesises evidence from 32 studies of individual-oriented interventions; that is, ones based on the strategy of supporting and improving potential employees (as distinct from ones that aim to improve the employment environment). These covered the three major national initiatives: the One Advisory Service, New Deal for Disabled People, and
Pathways to Work, and initiatives packaged with them (eg, Return to Work Credit, Permitted Work Rules, Condition Management Programme). There was evidence that personal advisors and individual case management in these schemes helped some participants back to work. However, these results were biased by widespread selection into these programmes of more work-ready claimants. Qualitative studies revealed the time pressures and requirements to fill job outcome targets that influenced the advisors' selection of claimants. Claimants were concerned about reduction in income and benefit sanctions that advisors could impose. This worked against the building up of mutual trust needed for the individual case management to work effectively.

Conclusions Selection into these programmes of more work ready claimants creates difficulties in judging to what extent the employment effects reported in the quantitative studies derive from the programmes or from the motivations of the individuals. We consider the implications for recent and future UK reforms of the finding that those furthest from the labour market need long-term engagement based on mutual trust to help them move towards work.

\section{BANKING CRISES AND MORTALITY DURING THE GREAT DEPRESSION: EVIDENCE FROM US URBAN POPULATIONS, 1929-1937}

doi:10.1136/jech.2010.120956.16

${ }^{1,2} \mathrm{D}$ Stuckler, ${ }^{3} \mathrm{C}$ Meissner, ${ }^{4} \mathrm{P}$ Fishback, ${ }^{5} \mathrm{~S}$ Basu, ${ }^{6} \mathrm{M}$ McKee. ${ }^{1}$ Department of Sociology, Oxford University, Oxford, UK; ${ }^{2}$ Department of Public Health and Policy, London School of Hygiene and Tropical Medicine, London, UK; ${ }^{3}$ Department of Economics and NBER, University of California, Davis, California, USA; ${ }^{4}$ Department of Economics and NBER, University of Arizona, Tucson, Arizona, USA; ${ }^{5}$ Department of Medicine and San Francisco General Hospital, Division of General Internal Medicine, University of California San Francisco, San Francisco, California, USA; ${ }^{6}$ London School of Hygiene and Tropical Medicine and European Observatory on Health Systems and Policies, London, UK

Objectives Previous research has suggested that the economic turmoil during the Great Depression led to significant improvements in public health. However, these studies have relied on highly aggregated national data (using less than 25 data points), and employed intermediary measures of economic change, such as employment and Gross Domestic Product. We use a new historical data set of US mortality rates and bank suspensions to analyse both the immediate and underlying causes of mortality change during Great Depression. Design Cause-specific mortality rates covering 114 US cities in 36 states were taken from the US Bureau of the Census. Bank suspensions data were taken from the Federal Deposit Insurance Corporation. Epidemiologic analysis was performed of the immediate causes of fluctuations in urban mortality rates weighted by population size. Dynamic fixed effects models were used to assess the immediate and delayed effects of bank suspensions on mortality. Setting 114 US cities and 36 US states, 1929-1937.

Participants NA.

Main outcome measure Age-standardised all-cause and cause-specific mortality rates

Results Reductions in all-cause mortality rates (about 10\% between 1929 and 1932) were attributable to declines in death rates due to pneumonia (26.4\% of total), influenza ( $13.1 \%$ of total), and respiratory tuberculosis $(11.2 \%$ of total), while death rates increased from heart disease (19.4\% of total), cancer $(8.1 \%$ of total) and diabetes $(2.9 \%)$. Of these main causes of mortality changes, only heart disease plausibly relates to contemporary economic shocks. A higher rate of bank suspensions was associated with contemporary higher suicide rates ( $\beta=0.32,95 \%$ CI 0.24 to 0.41 ) but lower death rates from motor vehicle accidents $(\beta=-0.18,95 \%$ CI -0.29 to $-0.07)$; no effect was observed for other causes of death studied. There was no evidence of substantially differing delayed effects. 